\title{
26455 - ASSESSMENT OF A NEW TECHNIQUE OF GLIDESCOPE INTUBATION
}

\section{Maria Angeles MD, Jenny Lam-McCulloch, MSc; Arsenio Avila, MD FRCPC; Sunnybrook And Women's College Health Sciences Centre, Toronto, ON, Canada}

INTRODUCTION: GlideScope is proven to be useful for difficult intubations. However, tracheal intubation using GlideScope takes a longer time compared to standard laryngoscopy.[1] This may be partially due to the fact that after exposing the larynx, additional time is needed to get the endotracheal tube (ETT) from the assistant and orient its view on the monitor. Another area of difficulty arises upon directing the ETT toward a clearly seen larynx. A modified way of GlideScope intubation is introduced in this study. This study aims to demonstrate that placement of ETT into the pharynx before the GlideScope blade facilitates faster and easier tracheal intubation.

METHODS: Following Ethics Board approval, patients scheduled for elective surgery under general anesthesia were recruited for the study. After informed consent, patients were randomized to one of two methods of tracheal intubation by GlideScope: (1) the conventional way of GlideScope laryngeal exposure followed by placement of ETT (control group); or (2) ETT placement into the pharynx prior to GlideScope laryngeal exposure (treatment group). Intubations were performed by two laryngoscopists with the same level of experience with the use of GlideScope. The time to successful intubation was measured in both groups by an independent observer. Time was measured from the moment the GlideScope blade or the ETT touched the patient's mouth and lasted until successful intubation was confirmed by the sustained presence of end-tidal $\mathrm{CO} 2$ on the capnograph. The difficulty with each mode of GlideScope intubation was assessed by an objective scoring system called the Intubation Difficulty Scale. A score of "0" indicates easy intubation and the degree of difficulty increases as the number increases to infinity. RESULTS: A total of 10 patients were recruited in this pilot study, 5 for each group. Time to successful intubation was slightly longer for the control group but not statistically significant ( $\mathrm{p}=0.17$ ). Similarly, the Intubation Difficulty Score for both groups were not statistically different $(\mathrm{p}=0.39)$.

DISCUSSION: Placement of ETT into the pharynx before the GlideScope blade may result in faster and easier intubation because initial view on the video monitor demonstrates the glottic opening and ETT at the same time. The ETT also serves to provide sufficient space for the entry of the GlideScope blade by sweeping the tongue away from the midline. Limiting time to successful intubation is beneficial in patients with limited pulmonary reserve. The sample size of this pilot study however, is too small to definitively state whether one method is superior to the other. The study team will continue to collect data over the coming months which will be added to this data. REFERENCES: [1] Br J Anaest 94: 381-384 
Table 1: Comparison of Two GlideScoge Techniques

\begin{tabular}{|c|c|c|}
\hline Technique & $\begin{array}{c}\text { Time to successful } \\
\text { intulbation (sec.) }\end{array}$ & $\begin{array}{c}\text { Intubation Difficulty } \\
\text { Score }(0-\square)\end{array}$ \\
\hline $\begin{array}{c}\text { GlideScope Larygneal Expostre } \\
\text { BEFORE ETT }\end{array}$ & $79.50 \pm 40.67$ & $0.75 \pm 0.96$ \\
\hline $\begin{array}{c}\text { ETT Phacement } \\
\text { BEFORE Laryngeal Exposure }\end{array}$ & $46.13 \pm 11.59$ & $0.25 \pm 0.50$
\end{tabular}

